\title{
AN OPD BASED PROSPECTIVE EXPLORATORY STUDY OF DERMATOPHYTOSIS - FOCUS ON TREATMENT OUTCOMES, MEDICATION ADHERENCE, AND QUALITY OF LIFE
}

\author{
SOUGATA MUKHERJEE ${ }^{1}$, CHIRANJIB BAGCHI ${ }^{2 *}$, KINGSHUK CHATTERJEE $^{3}$, AVIK DAS ${ }^{1}$, \\ SANTANU KUMAR TRIPATHI ${ }^{4}$
}

${ }^{1}$ Department of Pharmacology, Gupta College of Technological Sciences, Asansol, West Bengal, India. ${ }^{2}$ Department of Clinical and Experimental Pharmacology, School of Tropical Medicine, Kolkata, West Bengal, India. ${ }^{3}$ Department of Dermatology, School of Tropical Medicine, Kolkata, West Bengal, India. ${ }^{4}$ Department of Pharmacology, Netaji Subhas Medical College and Hospital, Bihar, Patna, India. Email: bchiranjib@yahoo.co.in

Received: 27 July 2021, Revised and Accepted: 02 August 2021

ABSTRACT

Objective: The study was planned primarily to judge the effectiveness, safety, and adherence of the prescribed treatments in dermatophytosis along with the effect on the Quality of Life (QoL) of the affected individuals.

Methods: In this prospective observational study, spanning over 5 months, participants (dermatophytosis patients) were assessed at baseline and 6 week-follow-up at study site. Information about their clinical presentation, QoL (Quality of Life) parameters, medication adherence, and adverse drug reaction (ADR) were entered in the Case Report Form (CRF) prepared by utilizing global standard tools such as 5D-itch scale, Dermatology Life Quality Index (DLQI), Medication Adherence Rating Scale (MARS), and PvPI-ADR reporting form respectively.

Results: Total 60 subjects (33 males and 27 females) had completed the study. Overall, tinea cruris (40\%) was the most common variety followed by combination of tinea corporis and cruris (35\%), tinea corporis (20\%), and tinea incognito (3\%). Pruritus was the predominant symptom. There was a significant $(\mathrm{p}<0.001)$ improvement of both 5D-itch \& DLQI scores from baseline to follow-up stage (after 6 weeks). Treatment regimens were well tolerated (only eight subjects reported any adverse event such as gastric irritation and sedation). Medication adherence to the prescribed treatment was good; (mean MARS score 7.588 \pm 1.82 ). Mostly prescribed regimen was a combination of antifungal (oral plus topical) and antihistamine tablet $(58 / 60,96.66 \%)$. The most common drug combination was capsule itraconazole, luliconazole cream, and cetirizine or levocetirizine tablet with or without ketoconazole soap $(35 / 60,58.33 \%)$.

Conclusion: Prescribed treatment regimen for dermatophytosis was effective, tolerable, acceptable with high medication adherence and also improved the QoL of the study subjects.

Keywords: Dermatophytosis, 5D-itch, Quality of life, Medication adherence, Treatment outcome, DLQI, Effectiveness.

(C) 2021 The Authors. Published by Innovare Academic Sciences Pvt Ltd. This is an open access article under the CC BY license (http://creativecommons.org/ licenses/by/4.0/) DOI: http://dx.doi.org/10.22159/ajpcr.2021v14i9.39239. Journal homepage: https://innovareacademics.in/journals/index.php/ajpcr

\section{INTRODUCTION}

Superficial fungal infections of hair, skin, and nails are the major cause of morbidity in India including West Bengal. Recently, dermatophytosis has emerged as an epidemic in many segments of India, posing a serious threat to our public health [1]. Dermatophytosis is a collective superficial fungal disease with a plethora of tinea manifestations, most frequently tinea cruris and tinea corporis. Trichophyton rubrum was reported as the leading fungal pathogen of tinea corporis and cruris in Rajasthan and Chennai whereas in Lucknow and Delhi,Trichophyton mentagrophytes and Microsporum audouinii were reported as predominant fungi. A recent publication from West Bengal had shown T. verrucosum, as the commonest causative organism thus displaying a varied and changing spectrum of the tinea infection over time $[1,2]$. Despite having a range of available anti-dermatophyte therapy, some treatment-related issues such as irrational use of antifungal agents, widespread use of cocktail antifungal-steroidal creams and suboptimal dose or duration of therapy often promote antifungal resistance and clinical failure. Other factors such as incorrect diagnosis, dressing pattern of the host including unhealthy lifestyle practices also provoke the recurrence of the disease [3-5].

While recalcitrant infections are becoming more and more prevalent, there is a relative paucity of literature revealing this changing scenario; hence, the present study.
Thus, the study was aimed to assess the overall effectiveness, tolerability, safety, and adherence to the treatment prescribed for dermatophytosis and its impact on the quality of life of the sufferers.

\section{MATERIALS AND METHODS}

Materials

This study was a self-responsive questionnaire based study, where standardized questionnaires were used for assessment of various parameters. For the assessment of clinical presentation, treatment outcome, QoL, tolerability, safety and medication adherence, we used 5D-Pruritus scale (Elman et al.), clinician perceived 5-Point Likert scale, DLQI questionnaire (Finlay et al.), PvPI-ADR reporting form, and MARS scale (Thompson et al.), respectively [6-11]. Besides, prescription pattern was assessed from served prescriptions and documented in the case report form (CRF).

Methods

Study design

This was a prospective observational single cohort study.

Study site and duration

This study was conducted at dermatology outpatient department (OPD) of a tertiary care teaching hospital, during November 2019 to 
March, 2020. Ethics approval was obtained from the Clinical Research Ethics Committee of the institute [Ref: CREC-STM/2019-AS12].

\section{Sample size}

All consecutive patients attending 2 outpatient days a week, freshlydiagnosed with dermatophytosis and having consented to take part in the study, were enrolled. Enrollment of study participants were done over one and half months starting from mid December 2019. Out of initially enrolled 114 study subjects, finally 60 subjects completed the follow-up visit after 6 weeks, the rest (54 subjects) did not turn up.

\section{Methodology}

Adult new patients of dermatophytosis (tinea infections of skin), clinically diagnosed and prescribed at the dermatology OPD were recruited as study subjects. After obtaining a written informed consent, study subjects were examined clinically and interviewed verbally. The diagnosis, treatment details, medication use history along with information on demographic and quality of life parameters were entered in the CRF and they were instructed to attend the OPD in the scheduled follow-up visit (after 6 weeks) as advised by the treating physician. In the follow-up visit, study subjects were again interviewed regarding their disease status, compliance of the medications, quality of life, treatment outcome etc. and data were entered in the CRF. In addition, physician's assessment about disease status and prescription details had been documented in the CRF.

\section{Parameters observed}

- Demographic characteristics at baseline

- Clinical presentation (at baseline and follow-up) and treatment outcome by $5 \mathrm{D}$ itch score and physician perceived 5 point Likert score

- QoL status (at baseline and follow-up) by DLQI score

- Adherence or compliance of the received treatment by MARS score.

\section{Statistical methods}

Data were collected and entered manually into Microsoft Excel 2016 and statistical analysis was done using SPSS version 20. Numerical data were expressed in Mean $\pm \mathrm{SD}$, categorical data in number (\%) and correlation analysis was done by Pearson's correlation coefficient. Distribution of the data was tested by prior test of normalcy (Kolmogorov-Smirnov Test).

\section{RESULTS}

Total 60 study subjects with dermatophytosis finally completed the study. Their demographic characteristics are depicted below (Table 1)

Regarding the treatment outcome, a significant reduction $(\mathrm{p}<0.01)$ of both 5D-itch (mean difference 7.467) and DLQI score (mean difference 6.850) from baseline to follow-up stage had been observed. As per clinician perceived effectiveness status (5-Point Likert score) majority, that is, $36(58.10 \%)$ appeared much better at follow-up stage after 6 weeks. While DLQI score was significantly improved from baseline

Table 1: Demographic characteristics of the study subjects

\begin{tabular}{lll}
\hline Sample size $(\mathbf{n})=\mathbf{6 0}$ & $\mathbf{n}(\mathbf{\%})$ & Mean $\mathbf{S D}$ \\
\hline Age (in years) & & $33.03 \pm 15.20$ \\
Age categorization & & \\
$\quad<35$ years & $36(60.00)$ & \\
$\geq 35$ years & $24(40.00)$ & \\
Gender & $33(55.00)$ & \\
$\quad$ Male & $27(45.00)$ & \\
Female & $12(20.00)$ \\
Diagnosis & $24(40.00)$ & \\
$\quad$ Tinea corporis & $21(35.00)$ \\
$\quad$ Tinea cruris & $3(5.00)$ & \\
Tinea corporis +Tinea cruris & \\
Tinea incognito & \\
\hline n (\%)=Number of subjects ( percentage), SD=Standard deviation
\end{tabular}

to follow-up, medication adherence had been found to be quite high $($ Mean \pm SD $=7.58 \pm 1.82), 34(56.66 \%)$ have high adherence (where adherence level considered as $\geq 8$ out of total MARS score) and $26(43.33 \%)$ shows poor adherence ( $<8$ out of total MARS score) but with minimum missed pill days (Mean $\pm \mathrm{SD}=2.87 \pm 4.66)$ (Table 2 and Fig. 1).

To test the strength of association between patient reported pruritus (5D-itch) with both QoL (DLQI) and physician perceived 5-point Likert score as well as between MARS score with both Missed dose and 5-Point Likert score, a correlation analysis (Pearson's correlation) was performed (Table 3).

The reported adverse events (AE) were few (only 8 out of 60 subjects) such as gastric irritation, sleep disturbance with itraconazole and sedation with cetirizine, levocetirizine (Table 4).

Most commonly prescribed regimen for dermatophytosis was found as a combination of antifungal (oral plus topical) and antihistamine tablet (58/60, 96.66\%). The most common drug combination was capsule itraconazole, luliconazole cream and cetirizine or levocetirizine tablet with or without ketoconazole soap $(35 / 60,58.33 \%)$. At follow-up visit,

Table 2: Statistics of the outcome parameters

\begin{tabular}{|c|c|c|c|c|}
\hline S. No. & Variable & n (\%) & Mean \pm SD & p value \\
\hline 1. & $\begin{array}{l}\text { 5D-itch Score } \\
\text { (at baseline) }\end{array}$ & & $17.63 \pm 2.93$ & $0.000 *$ \\
\hline 2. & $\begin{array}{l}\text { 5D-itch Score } \\
\text { (at follow-up) }\end{array}$ & & $10.17 \pm 3.57$ & \\
\hline \multirow[t]{6}{*}{3.} & $\begin{array}{l}\text { 5P-Likert Score } \\
\text { (at follow-up) }\end{array}$ & $4(6.50 \%)$ & $3.63 \pm 0.82$ & \\
\hline & Completely resolved (5) & & & \\
\hline & $\begin{array}{l}\text { Much better but still } \\
\text { present (4) }\end{array}$ & $36(58.10 \%)$ & & \\
\hline & $\begin{array}{l}\text { Little bit better but still } \\
\text { present (3) }\end{array}$ & $17(27.40 \%)$ & & \\
\hline & Unchanged (2) & $0(0 \%)$ & & \\
\hline & Getting worse (1) & $3(4.8 \%)$ & & \\
\hline 4. & $\begin{array}{l}\text { DLQI Score } \\
\text { (at baseline) }\end{array}$ & & $12.68 \pm 3.47$ & $0.000 *$ \\
\hline 5. & $\begin{array}{l}\text { DLQI Score (at } \\
\text { follow-up) }\end{array}$ & & $5.83 \pm 3.23$ & \\
\hline 6. & MARS Score & & $7.583 \pm 1.82$ & \\
\hline 7. & Missed dose(days) & & $2.87 \pm 4.66$ & \\
\hline 8. & Treatment Adherent & $34(56.66 \%)$ & & \\
\hline 9. & $\begin{array}{l}\text { Treatment Non- } \\
\text { Adherent }\end{array}$ & $26(43.33 \%)$ & & \\
\hline
\end{tabular}

$\mathrm{n}(\%)=$ number of subjects (in percentage), SD=Standard deviation, ${ }^{*}$ paired $\mathrm{t}$ test, Adherent: $\geq 8$ of total MARS score $\mathrm{n}(\%)=$ number of subjects

(in percentage), $\mathrm{SD}=\mathrm{Standard}$ deviation, *paired t test, Adherent: $\geq 8$ of total MARS score

Table 3: Correlation analysis between different assessment parameters

\begin{tabular}{llll}
\hline Variables & $\mathbf{n}$ & $\mathbf{p}$ & $\mathbf{r}^{* *}$ \\
\hline $\begin{array}{l}\text { 5D-Itch score (baseline) } \\
\text { DLQI score (baseline) }\end{array}$ & 60 & 0.000 & 0.502 \\
$\begin{array}{l}\text { 5D-Itch score (follow-up) } \\
\quad \text { DLQI score (follow-up) }\end{array}$ & 60 & 0.000 & 0.694 \\
$\begin{array}{l}\text { 5D-Itch diff (baseline-follow-up) } \\
\quad \text { DLQI diff (baseline-follow-up) }\end{array}$ & 60 & 0.000 & 0.531 \\
$\begin{array}{l}\text { 5D-itch diff (baseline-follow-up) } \\
\quad \text { 5-point Likert (follow-up) }\end{array}$ & 60 & 0.002 & 0.391 \\
$\begin{array}{l}\text { MARS score } \\
\quad \text { Missed dose (day) }\end{array}$ & 60 & 0.000 & -0.613 \\
$\begin{array}{l}\text { MARS score } \\
\text { 5-point Likert (at follow-up) }\end{array}$ & 60 & 0.009 & 0.337 \\
\hline **Pearson's correlation coefficient & & &
\end{tabular}




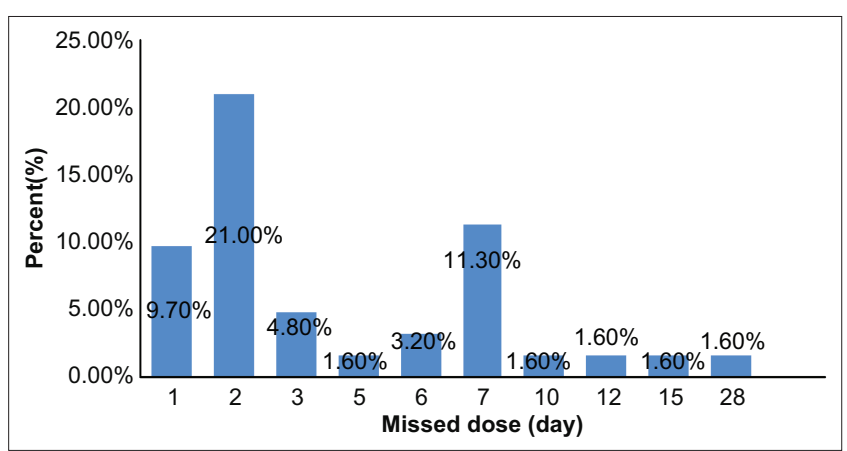

Fig. 1: Bar chart of missed dose of subjects

Table 4: Adverse event profile of the received treatment

\begin{tabular}{lllll}
\hline Adverse event & $\begin{array}{l}\text { Persons } \\
\text { reported }\end{array}$ & $\begin{array}{l}\text { Suspected } \\
\text { medication }\end{array}$ & $\begin{array}{l}\text { Dose } \\
\text { (mg) }\end{array}$ & $\begin{array}{l}\text { Frequency } \\
\text { (doses } \\
\text { consumed) }\end{array}$ \\
\hline Gastric irritation & 1 & Itraconazole & 200 & 1 \\
Sedation & 3 & Cetirizine & 10 & 1 \\
Sedation & 2 & Levocetirizine & 10 & 1 \\
Sleep disturbance & 1 & Itraconazole & 200 & 1 \\
Nocturnal emission & 1 & Itraconazole & 100 & 2 \\
\hline
\end{tabular}

we also studied the change of the initial regimen, altogether 30 (50\%) of prescriptions remained unchanged and were repeated. However, when changed, it happened between oral itraconazole and terbinafine, among topical luliconazole, sertaconazole, amorolfine, etc. Only in one subject all medicines were discontinued in first follow-up. All others received a second prescription though the disease was controlled substantially in them.

\section{DISCUSSION}

In the present study, tinea cruris was the most common site of manifestation followed by mixed (T. corporis and cruris) variety, tinea corporis, and tinea incognito (Table 1) whereas earlier studies showed tinea corporis as a predominant site of infection $[2,12]$. Mostly young population were affected (mean age 33 years) as they spend hours mostly in outdoor activities, similar trend was also seen in earlier studies. Pruritus/itching was the prime complaint in all types of dermatophytosis. Although it tends to be a mild symptom, it severely affects the patient's QoL. A previous study showed a positive correlation between itching and QoL parameter in dermatophytosis [13]. An appropriate treatment for dermatophytosis may have a great impact not only on clinical recovery but also in QoL improvement as evidenced by an earlier study [14]. In the present study, we also found a significant and moderately positive correlation between $5 \mathrm{D}$-itch and DLQI at both baseline and 6 weeks post-treatment follow-up stage. Our study had shown a significant $(\mathrm{p}<0.01)$ improvement in the clinical parameter (mean 5D-itch score) and QoL parameter (mean DLQI score) from baseline to follow-up visit at 6 weeks post treatment. Moreover, correlation between changes of 5D-itch and DLQI score from baseline to follow-up visit also showed a moderately positive correlation $(\mathrm{r}=0.531$, ${ }^{* *} \mathrm{p}<0.01$ ). So the prescribed treatment incurred a significant and favorable changes in both 5D-itch and DLQI score and thus was highly effective in dermatophytosis in this setting. As per clinician perceived effectiveness (by 5-Point Likert score), majority of study subjects (58.1\%) appeared much improved clinically in follow-up visit pending complete recovery and thus had been asked to continue the treatment further beyond 6 weeks while $6.5 \%$ achieved complete recovery (Table 2). We also tested the effectiveness on the basis of both clinician's judgment and patient's perception. Hence, the correlation between 5D-itch difference and 5-Point Likert score showed concordance (moderately positive correlation) of both patient's perception and clinician's interpretation of treatment effectiveness (Table 3).
Medication adherence has an immense role on treatment effectiveness. An earlier study on medication adherence among patients with tinea pedis was found to have low adherence to oral antifungal agents in higher percentage (59.6\%) [15]. But our study showed high adherence ( $\geq 80 \%$ of total MARS score) among majority of the study population $(56.66 \%)$ indicating a good compliance toward prescribed medications. Noteworthy, even among non-adherent population, $21 \%$ forgot to take medications for 2 days only whereas only $1.60 \%$ study subjects were non-adherent for more than 7 days (Fig. 1); so it reveals that adequate communication by health care providers fosters better treatment adherence and outcome (Table 3). Earlier study showed concomitant use of oral and topical antifungal agents increase medication adherence [15], the similar trend was also seen in the present study where a combination of antifungal (oral plus topical) and antihistamines was the predominantly prescribed regimen. Moreover, combination of oral itraconazole, luliconazole cream, and cetirizine or levocetirizine tablet with or without ketoconazole soap had been mostly prescribed, emerged as highly efficacious in this study. This is corroborating with a recently published study from the same region showing a changing pattern of infecting agent and drug sensitivity where itraconazole and luliconazole were found to be highly effective systemic and topical antifungals respectively against all Trichophyton species. [2] Earlier study on tolerability and safety of oral antifungals revealed common adverse events like nausea, rashes in 8-15\% patients (griseofulvin), gastrointestinal or skin related (terbinafine) in 10.5\% of population [16]. A favorable safety and tolerability profile of the treatment regimen was documented in the present study as noted above (Table 4).

Moreover, in most of the follow-up encounters (after 6 weeks as standard practice at study site), the primary prescriptions were repeated considering the favorable responses observed. Hence, corroborating with the changing pattern of the infecting organism and drug sensitivity, the standard treatment guideline and the procurement list of medicines should be updated at regular interval. The study had few limitations as well. Initially total 114 patients were recruited. However, in the follow-up all of them did not turn up. As it was a time bound (6 months) project, we could not recruit more subjects to adjust the loss to follow-up. Being a questionnaire based study; medication adherence was assessed by MARS (self-responsive tool) instead of any objective methods, but the subjective assessment of effectiveness (5D itch score) was corroborative with MARS score statistically, thus conforming to the patient's perception about disease status. Recurrence of disease is very common in dermatophytosis often because of persistence of infection and emerging resistance to the antifungal agents, facilitated by improper selection and inadequate duration of treatment. So a study with long follow-up can only throw a light on these issues.

\section{CONCLUSION}

The treatment provided for dermatophytosis at study site (Dermatology OPD of the tertiary care hospital) mostly comprised a combination regimen of antifungal (both oral and topical) and antihistamines, which was effective enough to foster the clinical improvement of the disease as well as quality of life of the study subjects, and the prescribed treatment regimen appeared safe, tolerable, rational, and acceptable with a good compliance.

\section{ACKNOWLEDGEMENT}

We sincerely acknowledge the contribution of the patients, physicians, all staff attending the dermatology OPD for their generous help toward successful execution of the study.

\section{AUTHORS' CONTRIBUTIONS}

Mr. Sougata Mukherjee contributed to the development of the protocol, conducted the research along with data collection, and authored the article. Dr. Chiranjib Bagchi contributed to the designing the study, intellectual content, protocol development for the conduct of the research, analysis of obtained data, and authored the article. 
Dr. Kingshuk Chatterjee contributed in designing, and clinical interpretation of the study data, authored the article. Mr. Avik Das contributed to the intellectual content, protocol development and authored the article. Dr. Santanu Kr Tripathi contributed to the intellectual content, designing the study, developing the protocol and authored the article.

\section{CONFLICT OF INTEREST}

Nil.

\section{AUTHORS FUNDING}

No specific funding was received by the authors.

\section{REFERENCES}

1. Sahoo AK, Mahajan R. Management of Tinea corporis, Tinea cruris, and Tinea pedis:A comprehensive review. Indian Dermatol Online J 2016;7:77-86

2. Das S, De A, Saha R, Sharma N, Khemka M, Singh S, et al. The current Indian epidemic of dermatophytosis:A study on causative agents and sensitivity patterns. Indian J Dermatol 2020;65:118-22.

3. Gandhi S, Patil S, Patil S, Badad A. Clinicoepidemiological study of dermatophyte infections in pediatric age group at a tertiary hospital in Karnataka. Indian J Paediatr Dermatol 2019;20:52-6.

4. Noronha TM, Tophakhane RS, Nadiger S. Clinico-microbiological study of dermatophytosis in a tertiary care hospital in North Karnataka. Indian Dermatol Online J 2016;7:264-71.

5. Dogra S, Uprety S. The menace of chronic and recurrent dermatophytosis in India: Is the problem deeper than we perceive? Indian Dermatol Online J 2016;7:73-6.

6. Rajashekar TS, Nandigonnanavar S, Kuppuswamy SK, Madhavi GS. Dermatology life quality index in patients with persisting and recurrent dermatophytosis. Int J Res Dermatol 2019;5:139-43.
7. Elman S, Hynan LS, Gabriel V, Mayo MJ. The 5-D itch scale:A new measure of pruritus. Br J Dermatol 2010;162:587-93.

8. Brown S. Likert Scale Examples for Surveys. Iowa State University, USA: ANR Program Evaluation; 2010. Available from: https://www. jordankmportal.com/resources/likert-scale-examples-for-surveys. [Last accessed on $2021 \mathrm{Apr} 25]$.

9. Finlay AY, Khan G. Dermatology life quality index (DLQI) a simple practical measure for routine clinical use. Clin Exp Dermatol 1994;19:210-6.

10. Standard format of Suspected Adverse Drug Reaction Reporting Form for Voluntary Reporting of Adverse Drug Reactions by Healthcare Professionals; 2020. Available from: http://www.ipc.nic.inor;http:// www.cdsco.nic.in/pharmacovigilance.htm. [Last accessed on 2021 Apr 25].

11. Thompson K, Kulkarni J, Sergejew AA. Reliability and validity of a new medication adherence rating scale (MARS) for the psychoses. Schizophr Res 2000;42:241-7.

12. Lakshmanan A, Ganeshkumar P, Mohan SR, Hemamalini M, Madhavan R. Epidemiological and clinical pattern of dermatomycoses in rural India. Indian J Med Microbiol 2015;33 Suppl:134-6.

13. Patro N, Panda M, Jena AK. The menace of superficial dermatophytosis on the quality of life of patients attending referral hospital in Eastern India: A cross-sectional observational study. Indian Dermatol Online J 2019;10:262.

14. Thomas J, Saple DG, Jerajani HR, Netha NR, Rangasamy DU, Shaikh $\mathrm{R}$, et al. Real-world, non-interventional, observational study of hydroxyzine hydrochloride in chronic pruritus:A prospective, noncomparative study. Dermatol Ther (Heidelb) 2019;9:299-308.

15. Tsunemi Y, Abe S, Kobayashi M, Kitami Y, Onozuka D, Hagihara A, et al. Adherence to oral and topical medication in 445 patients with tinea pedis as assessed by the Morisky medication adherence scale- 8 . Eur J Dermatol 2015;25:570-7.

16. Elewski B, Tavakkol A. Safety and tolerability of oral antifungal agents in the treatment of fungal nail disease:A proven reality.Ther Clin Risk Manag 2005;1:299-306. 